\title{
ON THE CLASS NUMBERS OF TOTALLY IMAGINARY QUADRATIC EXTENSIONS OF TOTALLY REAL FIELDS
}

\author{
BY JUDITH E. SUNLEY ${ }^{1}$ \\ Communicated by Oscar Goldman, June 22, 1971
}

\begin{abstract}
Let $K$ be an arbitrary totally real algebraic number field of degree $n \geqq 2$. It is shown that there exists an upper bound on the absolute value of the discriminant of any totally imaginary quadratic extension of $K$ of class number $h$ with at most one possible exception. This bound depends in an effective way on the parameters of the field $K$.
\end{abstract}

1. Introduction. The problem of determining all imaginary quadratic fields of class number $h$ has long been of importance in number theory. In recent years this problem has been solved for $h=1$ by Stark [8] and for $h=2$ by Goldstein [3], Baker [1], and Stark [9]. So far no real progress has been made for any other value of $h$.

This note is an announcement of research which extends some of the known results on class numbers of imaginary quadratic fields to the case of totally imaginary quadratic extensions of a totally real field. The main result is the following.

THEOREM 1. Let $K$ be an arbitrary totally real algebraic number field, $h$ an arbitrary positive integer. With at most one possible exception, all totally imaginary quadratic extensions $L$ of $K$ with class number $h$ satisfy

$$
\left|d_{L}\right|<C(K, h)
$$

where $C(K, h)$ is an effectively computable constant and $d_{L}$ is the discriminant of $L$.

This result is a generalization of similar theorems due to Heilbronn and Linfoot [4] for $h=1$ and Tatuzawa [11] for arbitrary $h$, both results in the special case where $K=\boldsymbol{Q}$ and $L$ is an imaginary quadratic field. A sketch of the proof will be given here. The details will appear elsewhere.

2. The estimate for $\Pi(x, \chi)$. Let $\Pi(x)=\sum_{N \mathfrak{N} \leqq x} 1$, and let $\Pi(x, \chi)=$ $\sum_{N \mathfrak{N} \leqq x} \chi(\mathfrak{U})$ where $\mathfrak{A}$ runs over all integral ideals of some algebraic number field $K$. In the case $K=\boldsymbol{Q}$ there is a classical result of Pólya [6] which says that $|\Pi(x, \chi)|<l^{1 / 2} \log l$, where $l$ is the period of the character $\chi$. At the same time Pólya's result appeared, Landau [5] obtained an extension of the result to the case where the degree of $K$ is at least two and $\chi$ is an ideal

AMS 1969 subject classifications. Primary 1065; Secondary 1068.

Key words and phrases. Number field, class number, imaginary quadratic extension.

1 This research was partially supported by NSF Science Development Grant GU-2061 and comprised the author's doctoral dissertation at the University of Maryland. 
character of $K$. Unfortunately, Landau's bound depends in an undetermined way on the parameters of the field $K$.

In the course of the proof of Theorem 1, the following effective form of Landau's theorem is obtained.

THEOREM 2 . Let $K$ be an algebraic number field of degree $n \geqq 2$. Let $\chi$ be any ideal character of $K$ which is primitive modulo its conductor $\mathfrak{\mathfrak { f }}$. Define $k=\left|N \mathfrak{f} \cdot d_{K}\right|$ where $N \mathfrak{f}$ is the norm of $\mathfrak{f}$ and $d_{K}$ is the discriminant of $K$. With $\Pi(x)$ and $\Pi(x, \chi)$ defined as above,

$$
\begin{gathered}
\left|\Pi(x)-\kappa_{K} x\right|<e^{28 \cdot 2 n+5}(n+1)^{5(n+1) / 2}\left|d_{K}\right|^{1 /(n+1)} \log ^{n}\left|d_{K}\right| \cdot x^{(n-1) /(n+1)} \\
|\Pi(x, \chi)|<e^{28 \cdot 2 n+6}(n+1)^{5 n / 2+7 / 2} k^{1 /(n+1)} \log ^{n} k \cdot x^{(n-1) /(n+1)}
\end{gathered}
$$

where $\kappa_{K}$ is the residue of $\zeta_{K}(s)$ at $s=1$.

In Schanuel's unpublished thesis [7] a similar bound is obtained using geometric methods rather than complex analysis. Again, however, the dependence of the constants involved on the parameters of the base field $K$ is not explicitly determined.

3. A lower bound for $L(1, \chi)$. The determination of a lower bound for $L(1, \chi)$ is the key element in the proof of Theorem 1 . It is in this determination that the possibility of an exception appears.

THEOREM 3. Let $K$ be any totally real algebraic number field of degree $n \geqq 2$ with $\chi$ a real, nonprincipal ideal character of $K$ primitive modulo its conductor $\mathfrak{f}$. Let $\varepsilon<\min \left\{1 /(n+1), 4(n+1)^{2} \alpha\right\}$ where $1-\alpha$ is the largest real zero of $\zeta_{K}(s)$. Assume $\log k \geqq \max \{4(n+11), 1 / \varepsilon\}$. Then

$$
L(1, \chi)>\frac{e^{-11 \cdot 443} \varepsilon^{(n+1) / 2}}{2^{n / 2} e^{n / 2}(n+1) \kappa_{K} k^{(n+2) \varepsilon / 2}}
$$

with at most one possible exceptional character $\chi$.

The proof of this theorem follows that of Tatuzawa [11] in the case $K=Q$ with ordinary Dirichlet characters replaced by ideal characters. The use of ideal characters makes the constants involved in the estimates considerably larger. The proof is based on the fact that when $L(1, \chi)$ is too small, $L(s, \chi)$ must have a real zero close to $s=1$. If $L\left(1, \chi_{1}\right)$ and $L\left(1, \chi_{2}\right)$ are both small and $\chi_{1} \chi_{2}$ not the principal character, then a certain analytic function would have too many zeros in a neighborhood of $s=1$ and a contradiction would be obtained. It should be noted that in Theorem 2 the order of magnitude of the estimates is $x^{(n-1) /(n+1)}$. It is crucial that the exponent of $x$ be less than one as that enables one to make estimates of the $L$-series in parts of the critical strip.

4. Proof of Theorem 1. The proof of Theorem 1 makes use of the above information on $L(1, \chi)$ and several facts about the relationship of the fields 
$K$ and $L$ where $K$ is totally real and $L$ is a totally imaginary quadratic extension of $K$. Let $\chi$ be the real ideal character of $K$ associated to $L$. Let $\mathfrak{f}$ be the conductor of $\chi$. Then the following facts apply :

a. $\zeta_{K}(s) L(s, \chi)=\zeta_{L}(s)$

b. $\kappa_{K} L(1, \chi)=\kappa_{L}$

c. the free part of the unit group of $L$ has the same rank as the free part of the unit group of $K$ and thus $2^{n-1} R_{K}=g_{0}(L / K) R_{L}$ where $R_{K}$ (respectively $R_{L}$ ) is the regulator of $K$ (respectively $L$ ), and $g_{0}(L / K)$ is a positive integer (see [2]);

d. $d_{L}=N \mathfrak{f} \cdot d_{K}^{2}$.

All these facts together with Theorem 3 imply Theorem 1 . The constant $C(K, h)$ can be shown to depend only on $h, d_{K}, h$, and the positive real zeros of $\zeta_{K}(s)$. For any given field $K$ this information is available and the constant is effectively determined.

\section{BIBLIOGRAPHY}

1. A. Baker, Imaginary quadratic fields of class number 2, Ann. of Math. (to appear).

2. L. Goldstein, A generalization of Stark's theorem, J. Number Theory (to appear).

3. Imaginary quadratic fields of class number 2, J. Number Theory (to appear).

4. H. Heilbronn and E. H. Linfoot, On the imaginary quadratic corpora of class number one, Quart. J. Math. Oxford Ser. 2 no. 5 (1934), 293-301.

5. E. Landau, Verallgemeinerung eines Polyaschen Satzes auf algebraische Zahlkörper, Göttinger Nachr. 1918, 478-488.

6. G. Pólya, Über die Verteilung der quadratischen Reste und Nichtreste, Göttinger Nachr. 1918, 21-29.

7. S. Schanuel, Heights in number fields, Unpublished Thesis, Columbia University, New York.

8. H. M. Stark, A complete determination of the complex quadratic fields of class-number one, Michigan Math. J. 14 (1967), 1-27. MR 36 \# 5102.

9. - Imaginary quadratic fields of class number 2, Ann. of Math. (to appear).

10. J. Sunley, On the class numbers of totally imaginary quadratic extensions of totally real fields, University of Maryland, College Park, Md., 1971.

11. T. Tatuzawa, On a theorem of Siegel, Japan J. Math. 21 (1951), 163-178. MR 14, 452. 20742

Department of Mathematics, University of Maryland, College Park, Maryland

Current address: Department of Mathematics and Statistics, American University, Washington, DC 20016 